\title{
A new comorbidities index for risk stratification for treatment of unruptured cerebral aneurysms
}

\author{
William C. Newman, MD, ${ }^{1}$ Dan W. Neal, MS, ${ }^{2}$ and Brian L. Hoh, MD² \\ ${ }^{1}$ Department of Neurological Surgery, University of Pittsburgh Medical Center, Pittsburgh, Pennsylvania; and ${ }^{2}$ Department of \\ Neurosurgery, University of Florida, Gainesville, Florida
}

\begin{abstract}
OBJECTIVE Comorbidities have an impact on risk stratification for outcomes in analyses of large patient databases. Although the Charlson Comorbidity Index $(\mathrm{CCl})$ and the Elixhauser Comorbidity Index (ECl) are the most commonly used comorbidity indexes, these have not been validated for patients with unruptured cerebral aneurysms; therefore, the authors created a comorbidity index specific to these patients.

METHODS The authors extracted all records involving unruptured cerebral aneurysms treated with clipping, coiling, or both from the Nationwide Inpatient Sample (2002-2010). They assessed the effect of 37 variables on poor outcome and used the results to create a risk score for these patients. The authors used a validation data set and bootstrapping to evaluate the new index and compared it to $\mathrm{CCl}$ and $\mathrm{ECl}$ in prediction of poor outcome, mortality, length of stay, and hospital charges.

RESULTS The index assigns integer values ( -2 to 7 ) to 20 comorbidities: neurological disorder, renal insufficiency, gastrointestinal bleeding, paralysis, acute myocardial infarction, electrolyte disorder, weight loss, metastatic cancer, drug abuse, arrhythmia, coagulopathy, cerebrovascular accident, psychosis, alcoholism, perivascular disease, valvular disease, tobacco use, hypothyroidism, depression, and hypercholesterolemia. Values are summed to determine a patient's risk score. The new index was better at predicting poor outcome than $\mathrm{CCl}$ or $\mathrm{ECl}$ (area under the receiver operating characteristic curve [AUC] 0.814 [95\% Cl 0.798-0.830], vs 0.694 and 0.712 , respectively, for the other indices), and it was also better at predicting mortality (AUC 0.775 [95\% $\mathrm{Cl} 0.754-0.792]$, vs 0.635 and 0.657 , respectively, for $\mathrm{CCl}$ and $\mathrm{ECl})$.

CONCLUSIONS This new comorbidity index outperforms the $\mathrm{CCl}$ and $\mathrm{ECl}$ in predicting poor outcome, mortality, length of stay, and total charges for patients with unruptured cerebral aneurysm. Reevaluation of other patient cohorts is warranted to determine the impact of more accurate patient stratification.
\end{abstract}

http://thejns.org/doi/abs/10.3171/2015.8.JNS14553

KEY WORDS unruptured cerebral aneurysm; comorbidity index; coiling; clipping; vascular disorders

$\mathrm{C}$ OMORBIDITIES impact patient stratification for outcome analyses in retrospective reviews in which large patient databases are used. For this reason, comorbidity stratification tools for outcome prediction are commonly used, such as the Charlson Comorbidity In$\operatorname{dex}(\mathrm{CCI})^{4}$ and the Elixhauser Comorbidity Index (ECI). ${ }^{8}$ These tools have been applied to a broad range of diseases and fields, including end-stage renal disease, ${ }^{5,6,11}$ pancreatic cancer, ${ }^{21}$ orthopedic surgery, ${ }^{15}$ general surgery, ${ }^{16}$ and neurovascular disease..$^{1,3,12,18}$ However, the broad range of diseases and fields to which these comorbidity indexes have been applied underscores their lack of specificity.
The CCI was designed for mortality analyses and derived from a small cohort of 604 patients with primary breast cancer at a single New York hospital. ${ }^{4}$ The ECI was derived from 438 acute-care hospitals in California, with a resultant set of 30 comorbidity measures with associated impacts on outcomes. ${ }^{8}$ Neither of these indexes was designed for use in unruptured cerebral aneurysms, but they have been applied to these patients. ${ }^{13,12,18}$ How accurately these indexes model the impact of disease burden in patients with unruptured cerebral aneurysms has not been studied.

Since the creation of the CCI in 1987, no studies have

ABBREVIATIONS AUC = area under the receiver operating characteristic curve; $\mathrm{CCI}=$ Charlson Comorbidity Index; $\mathrm{CVA}=\mathrm{cerebrovascular}$ accident; $\mathrm{ECI}=\mathrm{Elixhauser}$ Comorbidity Index; GEE = generalized estimating equations; GI = gastrointestinal; LOS = length of stay; MI = myocardial infarction; NIS = Nationwide Inpatient Sample; PHC4 = Pennsylvania Health Care Cost Containment Council.

SUBMITTED March 25, 2014. ACCEPTED August 12, 2015.

INCLUDE WHEN CITING Published online January 8, 2016; DOI: 10.3171/2015.8.JNS14553. 
been done to validate this tool in the neurosurgical patient population. Similarly, no formal study has been done to validate the ECI. Given the numerous medical and surgical advances that have occurred since their creation, there is a significant possibility that these older indices may overestimate the impact of patient comorbidities, thus confounding the results of current retrospective reviews.

It was not until recently that attention has been paid to the impact of preprocedural comorbidities on patient outcomes for ruptured and unruptured cerebral aneurysms. ${ }^{2}$ Using the Nationwide Inpatient Sample (NIS) database, Bekelis et al. were able to demonstrate the impact of 10 comorbidities on outcomes for ruptured and unruptured aneurysms undergoing clipping. However, their analysis did not take into account a full range of patient comorbidities and did not address the larger problem of designing a systematic way to incorporate these comorbidities into database analyses.

The NIS is a powerful database to study outcome data for cerebral aneurysms, and the question remains whether an index derived specifically from the disease population would outperform the CCI or ECI in mortality and morbidity prediction. Therefore, we set out to determine the impact of a wider range of comorbidities on patient outcomes for unruptured cerebral aneurysms undergoing treatment, and to use this information to create a comorbidity index applicable to this patient population. The implication of this study would be to propose an index more accurate than the CCI and ECI that should be used to analyze this patient population in all large patient databases.

\section{Methods}

The NIS database for the years 2002-2010 was obtained from the Agency for Healthcare Quality and Research's Healthcare Cost and Utilization Project (Rockville, MD). The NIS database is the largest all-payer hospital inpatient database in the US, comprising more than 7 million hospital stays and representing approximately 1000 hospitals each year. For each sampled hospital, all patient admissions for the year are recorded, allowing the calculation of annual hospital case volume. The years 2002-2010 were chosen because they represent the most up to date and homogeneously encoded data set available from the NIS. More information regarding the NIS database is provided at http://www.hcup-us.ahrq.gov/nisoverview.jsp.

The NIS database was queried for all patients 18 years or older with International Classification of Diseases, Ninth Revision (ICD-9) codes for the diagnosis of unruptured cerebral aneurysm (437.3) and procedure codes for either clipping (39.51), coiling $(39.79,39.72$, 39.52), or both (see Table 1 for patient demographic data). Patient comorbidities based on NIS or ICD-9 codes were then extracted for analysis (see Table 2 for comorbidity listings). Comorbidities were chosen based on a combination of the American Heart Association's perioperative cardiovascular risk factors ${ }^{9}$ and comorbid risk factors included in the $\mathrm{CCI}$ and ECI.

We used generalized estimating equations (GEE) to assess the effects of patient characteristics and comorbidities on the probability of poor outcome. Patients were classified as experiencing poor outcome if they died during admission or were discharged to hospice, to a skilled nursing, intermediate-care, or other long-term facility, or to a hospital-based Medicare-approved swing bed. Patients were classified as experiencing good outcome if they were discharged to home, to a short-term facility, or to an inpatient or outpatient rehabilitation facility, or if they left against medical advice. We assumed a binomial distribution for the outcome, and we included age, sex, and all comorbidities listed in Table 2 (excluding those associated with fewer than 5 cases of poor outcome) as potential predictors. To account for the clustering of observations on hospitals, we considered the hospital to be a repeated factor and assumed an exchangeable working correlation.

We assessed each model's predictive ability using the probability of concordance, or $c$ statistic, which is equal to the area under the receiver operating characteristic curve (AUC).

To evaluate how well the models might predict outcomes for a new group of patients, we used 2 validation methods. 1) We created a prediction model based on 2002-2008 NIS data (11,693 observations with complete information on all predictors) and used it to predict outcomes for patients recorded in the NIS database in 2009-2010 (4039

TABLE 1. Demographic data in patients with unruptured cerebral aneurysms recorded in the NIS*

\begin{tabular}{lcccc}
\hline & & \multicolumn{3}{c}{ Treatment } \\
\cline { 3 - 5 } Characteristic & Overall $(\mathrm{n}=18,423)$ & Clip $(\mathrm{n}=8,268)$ & Coil $(\mathrm{n}=9,834)$ & Both $(\mathrm{n}=321)$ \\
\hline Mean age in yrs, \pm SD (median) & $55.4 \pm 12.4(56.0)$ & $53.9 \pm 11.6(54.0)$ & $56.7 \pm 12.9(57.0)$ & $54.8 \pm 10.9(56.0)$ \\
\hline Sex (\% female) $\dagger$ & $13,758(75.0)$ & $6,135(74.5)$ & $7,383(75.5)$ & $240(74.8)$ \\
\hline Race† & & & \\
\hline White & $9,741(73.6)$ & $4,332(72.4)$ & $5,267(74.8)$ & $142(67.3)$ \\
\hline Black & $1,433(10.8)$ & $709(11.8)$ & $699(9.9)$ & $25(11.8)$ \\
\hline Hispanic & $1,327(10.0)$ & $621(10.4)$ & $677(9.6)$ & $29(13.7)$ \\
\hline Asian/Pacific Islander & $286(2.2)$ & $123(2.1)$ & $159(2.3)$ & $4(1.9)$ \\
\hline Native American & $59(0.4)$ & $36(0.6)$ & $20(0.3)$ & $3(1.4)$ \\
\hline Other & $386(2.9)$ & $163(2.7)$ & $215(3.1)$ & $8(3.8)$ \\
\hline * Except as otherwise noted, values are expressed as the number of patients (\%). & & \\
\hline
\end{tabular}


TABLE 2. Comorbidities in patients with unruptured cerebral aneurysms

\begin{tabular}{|c|c|c|c|c|}
\hline \multirow[b]{2}{*}{ Comorbidity* } & \multirow{2}{*}{$\begin{array}{c}\text { Overall } \\
(n=18,423)\end{array}$} & \multicolumn{3}{|c|}{ Treatment } \\
\hline & & Clip $(n=8268)$ & Coil $(n=9834)$ & Both $(n=321)$ \\
\hline AIDS & $22(0.1 \%)$ & $12(0.1 \%)$ & NR & $0(0 \%)$ \\
\hline Alcohol abuse & $473(2.6 \%)$ & $253(3.1 \%)$ & $204(2.1 \%)$ & $16(5.0 \%)$ \\
\hline Anemic deficiency & $1236(6.7 \%)$ & $608(7.4 \%)$ & $586(6.0 \%)$ & $42(13.1 \%)$ \\
\hline Arthritis & $442(2.4 \%)$ & $205(2.5 \%)$ & $225(2.3 \%)$ & $12(3.7 \%)$ \\
\hline Blood loss & $76(0.4 \%)$ & $32(0.4 \%)$ & $35(0.4 \%)$ & NR \\
\hline Chronic heart failure & $389(2.1 \%)$ & $177(2.1 \%)$ & $208(2.1 \%)$ & NR \\
\hline Chronic respiratory disease & $2966(16.1 \%)$ & $1356(16.4 \%)$ & $1560(15.9 \%)$ & $50(15.6 \%)$ \\
\hline Coagulopathy & $313(1.7 \%)$ & $144(1.7 \%)$ & $160(1.6 \%)$ & NR \\
\hline Depression & $1906(10.4 \%)$ & $886(10.7 \%)$ & $995(10.1 \%)$ & $25(7.8 \%)$ \\
\hline Diabetes w/o complications & $1716(9.3 \%)$ & $739(8.9 \%)$ & $954(9.7 \%)$ & $23(7.2 \%)$ \\
\hline Diabetes w/ complications & $99(0.5 \%)$ & $52(0.6 \%)$ & $44(0.4 \%)$ & NR \\
\hline Drug abuse & $337(1.8 \%)$ & $167(2.0 \%)$ & $158(1.6 \%)$ & $12(3.7 \%)$ \\
\hline Hypertension & $9993(54.2 \%)$ & $4495(54.4 \%)$ & $5314(54.0 \%)$ & $184(57.3 \%)$ \\
\hline Hypothyroidism & $1585(8.6 \%)$ & $670(8.1 \%)$ & $886(9.0 \%)$ & $29(9.0 \%)$ \\
\hline Liver disease & $218(1.2 \%)$ & $90(1.1 \%)$ & $123(1.3 \%)$ & NR \\
\hline Lymphoma & $30(0.2 \%)$ & $12(0.1 \%)$ & $17(0.2 \%)$ & NR \\
\hline Electrolyte disorder & $2185(11.9 \%)$ & $1210(14.6 \%)$ & $893(9.1 \%)$ & $82(25.6 \%)$ \\
\hline Metastatic cancer & $40(0.2 \%)$ & NR & $32(0.3 \%)$ & $0(0 \%)$ \\
\hline Neurological disorder & $243(1.3 \%)$ & $123(1.5 \%)$ & $103(1.1 \%)$ & $17(5.3 \%)$ \\
\hline Obesity & $873(4.7 \%)$ & $399(4.8 \%)$ & $464(4.7 \%)$ & NR \\
\hline Paralysis & $828(4.5 \%)$ & $484(5.9 \%)$ & $325(3.3 \%)$ & $19(5.9 \%)$ \\
\hline Perivascular disease & $738(4.0 \%)$ & $256(3.1 \%)$ & $461(4.7 \%)$ & $21(6.5 \%)$ \\
\hline Psychosis & $434(2.4 \%)$ & $223(2.7 \%)$ & $204(2.1 \%)$ & NR \\
\hline Pulmonary circulation disorder & $86(0.5 \%)$ & $39(0.5 \%)$ & $46(0.5 \%)$ & NR \\
\hline Renal failure & $326(1.8 \%)$ & $129(1.6 \%)$ & $191(1.9 \%)$ & NR \\
\hline Tumor w/o metastasis & $127(0.7 \%)$ & $55(0.7 \%)$ & $69(0.7 \%)$ & NR \\
\hline Peptic ulcer & $12(0.1 \%)$ & NR & NR & $0(0 \%)$ \\
\hline Valvular disease & $474(2.6 \%)$ & $220(2.7 \%)$ & $241(2.5 \%)$ & $13(4.1 \%)$ \\
\hline Weight loss & $193(1.0 \%)$ & $120(1.5 \%)$ & $59(0.6 \%)$ & $14(4.4 \%)$ \\
\hline Hypercholesterolemia & $3737(20.3 \%)$ & $1559(18.9 \%)$ & $2128(21.6 \%)$ & $50(15.6 \%)$ \\
\hline Cardiomyopathy & $119(0.6 \%)$ & $53(0.6 \%)$ & $64(0.7 \%)$ & NR \\
\hline Arrhythmia/dysrhythmia & $1270(6.9 \%)$ & $596(7.2 \%)$ & $657(6.7 \%)$ & $27(8.4 \%)$ \\
\hline CVA/TIA & $427(2.3 \%)$ & $209(2.5 \%)$ & $188(1.9 \%)$ & $30(9.3 \%)$ \\
\hline Angina pectoris & $104(0.6 \%)$ & $52(0.6 \%)$ & $50(0.5 \%)$ & NR \\
\hline Acute MI & $129(0.7 \%)$ & $66(0.8 \%)$ & $62(0.6 \%)$ & NR \\
\hline Old MI & $489(2.7 \%)$ & $215(2.6 \%)$ & $269(2.7 \%)$ & NR \\
\hline Renal insufficiency/acute kidney failure & $172(0.9 \%)$ & $79(1.0 \%)$ & $91(0.9 \%)$ & NR \\
\hline Chronic kidney disease & $179(1.0 \%)$ & $58(0.7 \%)$ & $118(1.2 \%)$ & NR \\
\hline GI bleeding & $26(0.1 \%)$ & NR & $17(0.2 \%)$ & NR \\
\hline Inflammatory bowel & $82(0.4 \%)$ & $31(0.4 \%)$ & $51(0.5 \%)$ & $0(0 \%)$ \\
\hline Leukemia & $14(0.1 \%)$ & NR & NR & $0(0 \%)$ \\
\hline Rheumatological & $269(1.5 \%)$ & $128(1.5 \%)$ & $138(1.4 \%)$ & NR \\
\hline Tobacco use & $4394(23.9 \%)$ & $2247(27.2 \%)$ & $2072(21.1 \%)$ & $75(23.4 \%)$ \\
\hline
\end{tabular}

NR = small number not reported.

* AIDS, blood loss, lymphoma, tumor, ulcer, angina, inflammatory bowel, and leukemia were excluded from consideration because of low incidence. Age and sex were also included in the model, but not listed in the table. 
complete observations). Although setting aside part of the original data as a validation data set generally results in less accurate prediction models,,$^{10}$ we included this step to evaluate how well a prediction model developed from NIS data might perform on a new data set. 2) We created a prediction model based on all available NIS data (2002-2010; 15,732 complete observations). To assess its likely predictive ability on a new data set, we bootstrapped the model 50 times, using the $c$ statistic to gauge the model's predictive ability for each bootstrapped sample. We report the mean and 95\% confidence interval of the bootstrapped $c$ statistics to provide an indication of how much variability in predictive ability we would expect to encounter if the model were used to predict outcomes for new patients.

To reduce the number of predictors so that the model might be more clinically useful, we used a stepwise elimination method based on the Akaike Information Criterion (i.e., the stepAIC function in the R software package MASS). Because stepwise procedures are known to be somewhat unstable and vulnerable to the influence of extreme observations,$^{10}$ we bootstrapped the stepwise routine 50 times and recorded the frequency with which each predictor was selected to remain in the final model. We considered a predictor selected in many bootstrap samples to be likely to perform better on a new data set than a predictor selected less often, and using this criterion, we took the top 20 predictors for poor outcome as our reduced model.

Finally, we used the coefficients of this model to create an integer-valued comorbidity index. We divided each coefficient by the absolute value of the smallest coefficient and rounded to the nearest integer. This established a point score for each comorbidity that varied between -2 (for hypercholesterolemia) to +7 (for neurological disorder). Our proposed comorbidity index is the sum of these integer values for each patient, a scale that varies from -5 to 55 . We used this scale to assign a comorbidity score to each patient in the 2002-2010 NIS data, and we then used this score and patient age as the only predictors of poor outcome, mortality, length of stay (LOS), and hospital charges in 4 GEE models. We used the $c$ statistic to assess the predictive ability of the comorbidity score, and we bootstrapped each model 50 times to estimate the performance of the comorbidity index as a predictor in a new data set.

For additional external validation, we then used the Pennsylvania Health Care Cost Containment Council (PHC4) database, a collection of all inpatient hospitalizations in Pennsylvania, for the fiscal years 2013-2014 (http://www.phc4.org/). We queried the database for all patients matching the previously noted inclusion and exclusion criteria. We then compared the predictive abilities of our neurovascular comorbidities index for the NIS and PHC4 database to predict mortality, poor outcome, and LOS; cost could not be compared because the PHC4 database does not include cost data elements.

\section{Results}

There were 18,423 total patients within the NIS database who met inclusion criteria for this study. Of these, 8268 underwent clipping, 9834 underwent coiling, and
321 underwent both treatments for their unruptured cerebral aneurysms. Of the 18,423 patients included, 405 died after treatment (2.2\% overall mortality rate), findings in line with those of Raaymakers, et al. ${ }^{19}$ On querying the PHC4 database, we found there were 1090 patients who met inclusion criteria, with a similar distribution of clipping and coiling.

\section{Significant Comorbidities}

Multivariate analysis of 37 of the 43 patient comorbidities listed in Table 2 found the following variables to be significant predictors of poor outcome: age, neurological disorder, renal insufficiency, gastrointestinal (GI) bleeding, paralysis, acute myocardial infarction (MI), electrolyte disorder, weight loss, metastatic cancer, drug abuse, arrhythmia, coagulopathy, cerebrovascular accident (CVA), psychosis, alcoholism, perivascular disease, tobacco use, hypothyroidism, depression, and hypercholesterolemia ( $\mathrm{p}<0.05$ for all).

\section{Predictive Model}

The statistically significant comorbidities were weighted based on their relative impact on patient outcomes to create a comorbidity index (see Table 3 for results). Individual scores ranged from -2 to 7 , with positive scores denoting increased odds of poor outcome and negative scores denoting an inverse relationship with poor outcome.

To calculate a patient's comorbidity score, all individual comorbidity scores must be added. For example, a patient with renal insufficiency (+6) and CVA (+2) would

\section{TABLE 3. Comorbidity scoring index}

\begin{tabular}{lcc}
\hline \multicolumn{1}{c}{ Comorbidity } & Point Score & p Value \\
\hline Neurological disorder & 7 & $<0.001$ \\
\hline Renal insufficiency & 6 & $<0.001$ \\
\hline Gl bleeding & 6 & $<0.05$ \\
\hline Paralysis & 5 & $<0.001$ \\
\hline Acute Ml & 5 & $<0.001$ \\
\hline Electrolyte disorder & 4 & $<0.001$ \\
\hline Weight loss & 4 & $<0.001$ \\
\hline Metastatic cancer & 4 & $<0.01$ \\
\hline Drug abuse & 3 & $<0.001$ \\
\hline Arrhythmia & 2 & $<0.001$ \\
\hline Coagulopathy & 2 & $<0.01$ \\
\hline CVA & 2 & $<0.001$ \\
\hline Psychosis & 2 & $<0.01$ \\
\hline Alcoholism & 1 & $<0.05$ \\
\hline Perivascular disease & 1 & $<0.01$ \\
\hline Valvular disease & 1 & $<0.05$ \\
\hline No comorbidities & 0 & $\mathrm{NA}$ \\
\hline Tobacco use & -1 & $<0.001$ \\
\hline Hypothyroidism & -1 & $<0.01$ \\
\hline Depression & -1 & $<0.01$ \\
\hline Hypercholesterolemia & -2 & $<0.001$ \\
\hline
\end{tabular}

$\mathrm{NA}=$ not applicable. 
have a total score of +8 . A patient with history of tobacco use $(-1)$ and valvular disease $(+1)$ would have a score of 0 . A patient with hypercholesterolemia $(-2)$ and tobacco use $(-1)$ would have a score of -3 . The sum score can then be used to stratify patients based on their preoperative comorbidities, the same as with the CCI and ECI. See Fig. 1 for the distribution of index scores.

Of note, to make this model comparable to the $\mathrm{CCI}$ and ECI, we did not assign a point value to age. It should be noted that increasing age was associated with an increasing mortality rate and poor outcome. Age should be used in conjunction with our scoring index to best stratify risk in patients.

\section{Accuracy of the Model}

We compared the performance of our model against the CCI in predicting mortality, poor outcome, LOS, and hospital costs because these are results for which the ECI and CCI are commonly used. ${ }^{1,3,12,18}$ To compare our index to the CCI and ECI as a predictor of mortality and poor outcome, we used the $c$ statistic, a measure of how well a model can discriminate between subjects experiencing an event and not experiencing an event. The $c$ statistic is equal to the AUC, with $c=1$ indicating perfect prediction and $c=0.5$ indicating no predictive ability.

A GEE model with our neurovascular comorbidities index and age as the only covariates was a highly significant predictor of mortality $(\mathrm{p}<0.001$ ) for patients with unruptured cerebral aneurysms in the 2002-2010 NIS data. The model had a $c$ statistic of 0.775 . In 50 bootstrap iterations, the mean $c$ statistic was 0.773 (95\% CI 0.754-0.792), suggesting that our index's predictive ability would remain consistent for other groups of patients with unruptured aneurysms. Similar models using CCI and ECI in place of our index had $c$ statistics of 0.635 and 0.657 , respectively, when used to predict outcomes for 2002-2010 NIS data. This demonstrates the significantly superior ability of our index to predict mortality compared with that of the CCI and ECI.

For predicting poor outcome, a model with our index and age as the only covariates was highly significant $(\mathrm{p}<$ 0.001 ) and had a $c$ statistic of 0.814 . In 50 bootstrap runs, the mean $c$ statistic was also 0.814 (95\% CI 0.798-0.830). Similar models using CCI and ECI in place of our index had $c$ statistics of 0.694 and 0.712 , respectively.

To assess the indices' abilities to predict LOS and total hospital charges, we categorized the outcomes and compared the proportion of patients who were correctly classified by each index. For LOS, we compared the ability of each index to predict a short stay ( $<6$ days), medium stay (6-14 days), or long stay ( $>14$ days). A model with our index and age as the only predictors correctly classified $69.5 \%$ of patients in 2002-2010 NIS data, compared with $63 \%$ for the CCI and $61.4 \%$ for the ECI $(61.4 \%)$. None of the indices performed well in classifying patients who experienced long stays, but although the accuracy of the CCI and ECI for predicting this category was near 0 , our index captured $18 \%$ of these patients (see Table 4 for details).

For comparing the indices' abilities to predict costs, we categorized charges as low, low to medium, medium to high, and high according to the quartiles of the distribu-

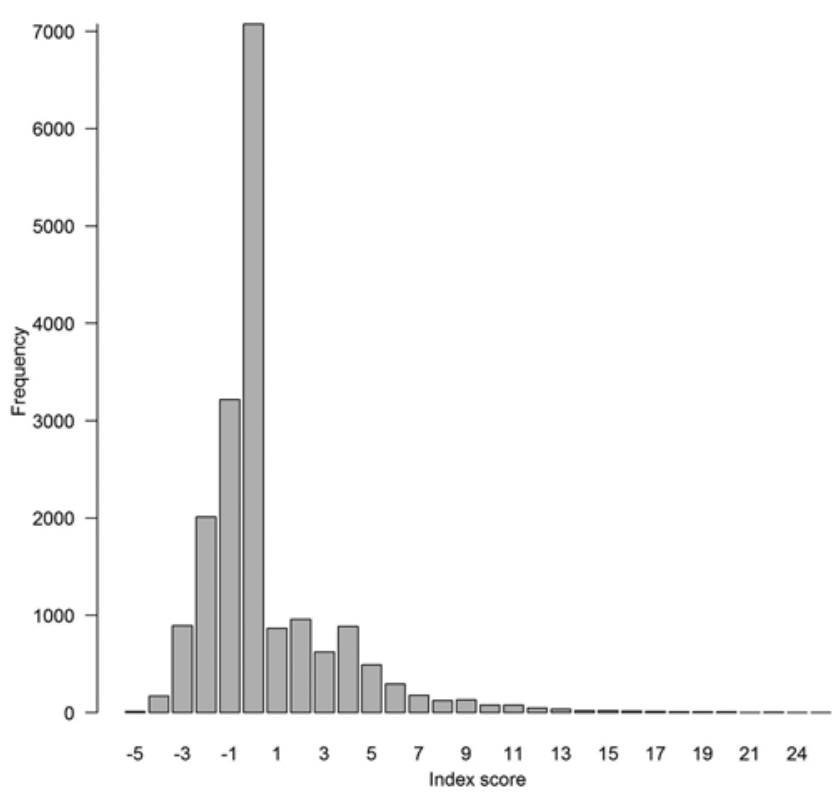

FIG. 1. Bar graph depicting distribution of patient comorbidity scores for the neurovascular comorbidity index. The values on the $x$ axis represent the total patient comorbidity index score.

tion of hospital charges in the 2002-2010 NIS data, and we evaluated each index's ability to correctly classify patients (see Table 4 for details). All indices were poor predictors of costs. A model with our index and age as the only covariates demonstrated an overall accuracy of only $31 \%$, compared with $25.8 \%$ for the CCI and $27 \%$ for the ECI (see Table 4 for details).

For external validation, we analyzed the ability of our neurovascular index to predict mortality, poor outcome, and LOS using the PHC4 database. We found that our neurovascular index performed similarly well with the PHC4 database compared with its performance in the NIS database. Concerning mortality, our neurovascular index demonstrated a $c$ statistic of 0.775 , slightly better than its performance in the NIS database. Regarding poor outcome, our neurovascular index had a $c$ statistic of 0.814 , not significantly different than its value of 0.814 in the NIS database. For LOS, our index demonstrated an accuracy of $89.8 \%$ for predicting short, $33.5 \%$ for medium, and $17.9 \%$ for long hospitalizations.

\section{Discussion}

The identification of preoperative risk factors and the ability to stratify patients based on their risk is the cornerstone of large database reviews. Until recently, much of the literature has used the CCI or the ECI for patient stratification. ${ }^{1,3,5,6,11,12,15,16,18,21}$ However, groups such as Liu et al. have demonstrated the inferiority of general indexes, specifically the CCI, to more disease-specific indexes in their paper on outcomes in dialysis patients. ${ }^{17}$ Similarly, neurovascular diseases are in need of a more disease-specific model for patient stratification.

Our neurovascular index for unruptured cerebral aneurysms receiving clipping or coiling was compared with 
TABLE 4. Comparison of $\mathrm{CCI}, \mathrm{ECl}$, and our neurovascular comorbidities index from 2002 to 2010

\begin{tabular}{llll}
\hline \multicolumn{1}{c}{ Variable } & Our Index +Age & \multicolumn{1}{c}{$\mathrm{CCl}+$ Age } & ECl + Age \\
\hline Mortality & \multicolumn{1}{c}{$c=0.775$} & $c=0.635$ & $c=0.657$ \\
\hline Poor outcome & \multicolumn{1}{c}{$c=0.814$} & $c=0.694$ & $c=0.712$ \\
\hline LOS (correct categorizations) ${ }^{*}$ & Overall: $69.5 \%$ & Overall: $63.0 \%$ & Overall: $61.4 \%$ \\
& Short stays: $89.8 \%$ & Short stays: $86.5 \%$ & Short stays: $79.8 \%$ \\
& Medium stays: $33.5 \%$ & Medium stays: $22.9 \%$ & Medium stays: $35.7 \%$ \\
& Long stays: $17.9 \%$ & Long stays: $0.25 \%$ & Long stays: $0.35 \%$ \\
\hline Costs (correct categorizations) $\dagger$ & Overall: $31.0 \%$ & Overall: $25.8 \%$ & Overall: $27.0 \%$ \\
& Low costs: $0.1 \%$ & Low costs: $0 \%$ & Low costs: $0 \%$ \\
& Low/medium: $66.2 \%$ & Low/medium: $25.5 \%$ & Low $/$ medium: $54.5 \%$ \\
& Medium/high: $35.0 \%$ & Medium/high: $76.4 \%$ & Medium $/$ high: $50.4 \%$ \\
& High: $19.3 \%$ & High: $0.9 \%$ & High: $1.0 \%$ \\
\hline
\end{tabular}

* Short stays, $<6$ days; medium stays, $6-14$ days; long stays, $>14$ days.

† Low costs, < $\$ 45,000$; low/medium costs, $\$ 45,001-\$ 69,000$; medium/high costs, $\$ 69,001-\$ 113,000$; high costs, $>\$ 113,000$.

the CCI and ECI with respect to mortality, poor outcome, LOS, and costs. Our model demonstrated a marked improvement in prediction for all 4 outcomes, particularly poor outcome and mortality, without a significant increase in the number of variables incorporated into the model.

Similar results were seen when we applied our neurovascular index to the PHC4 database: prediction of mortality and poor outcome maintained levels of accuracy as high as those seen in the NIS database. Although the ability to predict LOS was worse with the PHC4 database, the average LOS in the NIS database was significantly shorter than in the PHC4 database (6.4 days vs 3.9 days, respectively). However, neither our neurovascular index nor the CCI or ECI demonstrated good predictive abilities for LOS.

The most significant factors affecting patient outcomes were neurological disorder, renal insufficiency, GI bleeding, paralysis, and acute MI. Neurological disorder may carry such a significant weight because it may be a surrogate marker for cerebral aneurysm size, with larger aneurysms producing neurological deficits due to mass effect. In their meta-analysis, Raaymakers et al. found aneurysm size to be strongly predictive of patient outcomes. ${ }^{19}$ Similarly, aneurysms of sufficient size could produce paralysis as a manifestation of their neurological deficit.

Renal insufficiency, acute MI, arrhythmias, valvular disease, perivascular disease, and CVA also conferred significant risk of poor outcome. This is not unexpected; the recent American Heart Association guidelines for perioperative cardiovascular risk for noncardiac surgery listed these preoperative comorbidities as significant predictors of perioperative cardiac events. ${ }^{9}$

Other disorders such as GI bleeding, coagulopathy, metastatic cancer, and significant weight loss do not have firm support in the literature for their effects on postoperative mortality in unruptured cerebral aneurysms. It is possible that these disorders are surrogate markers for overall patient health status and that their presence implies less ability to tolerate procedural interventions. Further investigation into the role of these comorbidities is warranted.

Interestingly, hypercholesterolemia and tobacco use were negative predictive factors for mortality. The seemingly protective effect of hypercholesterolemia could be due to the potential benefits of statin therapy in unruptured cerebral aneurysms. ${ }^{20}$

Concerning tobacco use, Eftekhar and Morgan demonstrated no significant impact of tobacco use on outcomes after surgical treatment of unruptured aneurysms. ${ }^{7}$ Moreover, Juvela demonstrated that although smoking may carry an increased risk of subarachnoid hemorrhage ( $\mathrm{p}<$ 0.01 ), there is not an associated increase in mortality rate after subarachnoid hemorrhage. ${ }^{13}$ Tobacco use has, however, been linked to increased aneurysm formation. ${ }^{14}$ Taken together, the seemingly protective nature of tobacco use may reflect its ability to bring this population of people to earlier screening, detection, and intervention based on their known risk factor.

The absence of diabetes and hypertension as significant predictors of patient outcomes is also an interesting aspect of our index. This may be the result of the confounding effects of the end organ complications of these diseases. For example, hypertension and diabetes are linked to renal insufficiency, acute MI, perivascular disease, and CVA, all of which are significant predictors of poor outcome in our study. Although hypertension and diabetes are known to be associated with poor outcomes in general, their predictive power may be overshadowed in our analysis by their end effects, which were included in our models and which we found to have even stronger associations with poor outcome.

Many groups are using the NIS database to study cerebral aneurysms. Our neurovascular index demonstrates superior performance compared with the CCI or ECI. Therefore, future studies should make use of our index for stratifying patients with unruptured cerebral aneurysms.

\section{Study Limitations}

There are several important limitations to this study. In using the NIS database, coding inaccuracies are inevitable, which can lead to a skewing of results. During our period of study from 2002 to 2010 there was a uniform set of codes for the procedures analyzed, and our methodology has been consistent with the published literature,,$^{1,3,12,18}$ but this does not eliminate data entry inaccuracies. Another limitation is the lack of data regarding aneurysm charac- 
teristics in the NIS database. The NIS does not contain data about location, size, or geometry, which impact surgical decision making as well as patient outcomes. Additionally, the retrospective nature of our study introduces a degree of selection bias inherent to all retrospective reviews.

Another limitation of this study is our inability to use the modified Rankin Scale, a commonly used scale for disability. Information regarding pre- and postoperative disability is not available within the NIS database, and we therefore used discharge status as a surrogate marker for postoperative functional status. However, this limitation is seen throughout retrospective administrative database analyses.

\section{Conclusions}

Large database reviews need accurate methods for patient stratification to produce the most accurate results. We created a novel neurovascular comorbidity index for unruptured cerebral aneurysms, which significantly outperforms both the CCI and the ECI in predicting mortality, poor outcome, LOS, and costs. The index's improved predictive abilities suggest that perhaps it should be used in preference to the CCI or ECI for risk stratification in patients with unruptured cerebral aneurysms. Reevaluation of other patient cohorts is warranted to determine the impact of more accurate patient stratification on studies.

\section{References}

1. Bekelis K, Missios S, Labropoulos N: Regional and socioeconomic disparities in the treatment of unruptured cerebral aneurysms in the USA: 2000-2010. J Neurointerv Surg 6:556-560, 2014

2. Bekelis K, Missios S, Mackenzie TA, Desai A, Fischer A, Labropoulos, et al: Predicting inpatient complications from cerebral aneurysm clipping: the Nationwide Inpatient Sample 2005-2009. J Neurosurg 120:591-598, 2014

3. Brinjikji W, Rabinstein AA, Lanzino G, Cloft HJ: Racial and ethnic disparities in the treatment of unruptured intracranial aneurysms: a study of the Nationwide Inpatient Sample 2001-2009. Stroke 43:3200-3206, 2012

4. Charlson ME, Pompei P, Ales KL, MacKenzie CR: A new method of classifying prognostic comorbidity in longitudinal studies: development and validation. J Chronic Dis 40:373383, 1987

5. Collins AJ, Weinhandl E, Snyder JJ, Chen SC, Gilbertson D: Comparison and survival of hemodialysis and peritoneal dialysis in the elderly. Semin Dial 15:98-102, 2002

6. Di Iorio B, Cillo N, Cirillo M, De Santo NG: Charlson Comorbidity Index is a predictor of outcomes in incident hemodialysis patients and correlates with phase angle and hospitalization. Int J Artif Organs 27:330-336, 2004

7. Eftekhar B, Morgan MK: Preoperative factors affecting the outcome of unruptured posterior circulation aneurysm surgery. J Clin Neurosci 18:85-89, 2011

8. Elixhauser A, Steiner C, Harris DR, Coffey RM: Comorbidity measures for use with administrative data. Med Care 36:8-27, 1998

9. Fleisher LA, Beckman JA, Brown KA, Calkins H, Chaikof E, Fleischmann KE, et al: ACC/AHA 2007 Guidelines on Perioperative Cardiovascular Evaluation and Care for Noncardiac Surgery: executive summary. Circulation 116:1971-1996, 2007
10. Harrell FE Jr: Regression Modeling Strategies. New York: Springer, 2001

11. Hemmelgarn BR, Manns BJ, Quan H, Ghali WA: Adapting the Charlson Comorbidity Index for use in patients with ESRD. Am J Kidney Dis 42:125-132, 2003

12. Higashida RT, Lahue BJ, Torbey MT, Hopkins LN, Leip E, Hanley DF: Treatment of unruptured intracranial aneurysms: a nationwide assessment of effectiveness. AJNR Am J Neuroradiol 28:146-151, 2007

13. Juvela S: Prehemorrhage risk factors for fatal intracranial aneurysm rupture. Stroke 34:1852-1857, 2003

14. Juvela S, Poussa K, Porras M: Factors affecting formation and growth of intracranial aneurysms: a long-term follow-up study. Stroke 32:485-491, 2001

15. Kos FT, Yazici O, Civelek B, Seker M, Arik Z, Aksoy S, et al: Evaluation of the effect of comorbidity on survival in pancreatic cancer by using "Charlson Comorbidity Index" and "Cumulative Illness Rating Scale." Wien Klin Wochenschr 126:36-41, 2014

16. Letarte F, Hallet J, Drolet S, Charles Grégoire R, Bouchard A, Gagné JP, et al: Laparoscopic emergency surgery for diverticular disease that failed medical treatment: a valuable option? Results of a retrospective comparative cohort study. Dis Colon Rectum 56:1395-1402, 2013

17. Liu J, Huang Z, Gilbertson DT, Foley RN, Collins AJ: An improved comorbidity index for outcome analyses among dialysis patients. Kidney Int 77:141-151, 2010

18. McDonald RJ, Cloft HJ, Kallmes DF: Intracranial hemorrhage is much more common after carotid stenting than after endarterectomy: evidence from the National Inpatient Sample. Stroke 42:2782-2787, 2011

19. Raaymakers TW, Rinkel GJ, Limburg M, Algra A: Mortality and morbidity of surgery for unruptured intracranial aneurysms: a meta-analysis. Stroke 29:1531-1538, 1998

20. Valença MM: "Sit back, observe, and wait." Or is there a pharmacologic preventive treatment for cerebral aneurysms? Neurosurg Rev 36:1-10, 2013

21. Voskuikl T, Hageman M, Ring D: Higher Charlson Comorbidity Index scores are associated with readmission after orthopaedic surgery. Clin Orthop Relat Res 472:1638-1644, 2014

\section{Disclosures}

The authors report no conflict of interest concerning the materials or methods used in this study or the findings specified in this paper.

\section{Author Contributions}

Conception and design: Hoh, Newman. Acquisition of data: Newman, Neal. Analysis and interpretation of data: all authors. Drafting the article: all authors. Critically revising the article: all authors. Reviewed submitted version of manuscript: all authors. Statistical analysis: Neal. Study supervision: Hoh.

\section{Supplemental Information}

\section{Previous Presentations}

Portions of this work were presented in abstract and poster form at the AANS 2014 Annual Meeting in San Francisco, California, which was held on April 5-9, 2014.

\section{Correspondence}

Brian L. Hoh, Department of Neurosurgery, University of Florida, P.O. Box 100265, Gainesville, FL 32610. email: brian.hoh@ neurosurgery.ufl.edu. 